\title{
Procesos de resiliencia comunitaria en situación de desastres: estudio de caso de la comunidad de Mapachico Centro de Pasto (Colombia) ${ }^{4}$
}

\author{
Freddy Arley Ruano Bermúdez \\ Psicólogo \\ Universidad de Nariño, Colombia \\ Hospital Infantil Los Ángeles de Pasto, Colombia \\ Correo electrónico: freddyarleyr@gmail.com \\ Paula Alejandra Riascos $\begin{array}{r}\text { Yandar } \\ \text { Psicóloga }\end{array}$
Universidad de Narino, Colombia
Correo electrónico: paulaalejandra@udenar.edu.co
}

Katherine Alejandra Castillo

Egresada de Psicología

Universidad de Nariño, Colombia

Correo electrónico: kathaleja05@gmail.com
Recibido: 02/04/2020

Evaluado: 09/06/2020

Aceptado: 17/06/2020

\section{Resumen}

La resiliencia comunitaria es un constructo novedoso que permite reconocer el papel activo que presentan las comunidades al momento de enfrentar una adversidad 0 afectación colectiva. Es por eso que el presente artículo de investigación tiene como objetivo analizar el papel de la resiliencia comunitaria frente a los desastres en un grupo de habitantes de la comunidad de Mapachico Centro de la ciudad de Pasto. La investigación se desarrolló desde el paradigma cualitativo, el enfoque fenomenológico y el tipo de estudio etnometodológico. Los resultados evidencian que los componentes de autoestima colectiva, identidad cultural y solidaridad favorecen la reconstrucción del tejido social y configuran las percepciones frente al riesgo, mientras que el humor social se perfila como una oportunidad para generar procesos de resistencia comunitaria. Se concluye que la resiliencia comunitaria se expresa como una característica que permite la integración e identificación de los miembros en temas de desarrollo social.

Resiliencia, volcán, desastre natural, comunidad, salud mental.

4 Para citar este artículo: Ruano, F., Riascos, P. y Castillo, K. (2022). Procesos de resiliencia comunitaria en situación de desastres: estudio de caso de la comunidad de Mapachico Centro de Pasto (Colombia). Informes Psicológicos, 22(1), pp. 77-95 http://dx.doi.org/10.18566/infpsic.v22n1a04 


\section{Community resilience processes in disaster situations: case study of the Mapachico community in the center of Pasto (Colombia)}

Abstract

Community resilience is a novel construct that allows us to recognize the active role that communities play when facing adversity or collective affectation. That is why this research article aims to analyze the role of community resilience in the face of disasters in a group of inhabitants of the Mapachico community in the center of the city of Pasto. The research was developed from the qualitative paradigm, the phenomenological approach and the type of ethnomethodological study. The results show that the components of collective self-esteem, cultural identity and solidarity favor the reconstruction of the social fabric and shape perceptions of risk, while social humor is emerging as an opportunity to generate community resistance processes. As a conclusion, it can be said that community resilience is expressed as a characteristic that allows the integration and identification of members in social development issues.

Keywords

Resilience, volcano, natural disaster, community, mental health.

\section{Processos de resiliência comunitária em situações de desastre: estudo de caso da comunidade de Mapachico Centro de Pasto (Colômbia)}

Resumo

A resiliência da comunidade é uma construção nova que nos permite reconhecer o papel ativo que as comunidades desempenham quando enfrentam adversidades ou afetação coletiva. É por isso que este artigo de pesquisa tem como objetivo analisar o papel da resiliência da comunidade frente aos desastres em um grupo de habitantes da comunidade Mapachico no centro da cidade de Pasto. A pesquisa foi desenvolvida a partir do paradigma qualitativo, da abordagem fenomenológica e do tipo de estudo etnometodológico. Os resultados mostram que os componentes de autoestima coletiva, identidade cultural e solidariedade favorecem a reconstrução do tecido social e configuram percepções de risco, enquanto 0 humor social surge como oportunidade de gerar processos de resistência comunitária. Concluindo que a resiliência da comunidade se expressa como uma característica que permite a integração e identificação dos membros nas questões de desenvolvimento social. 


\section{ntroducción}

Colombia, dadas sus condiciones geográficas, geológicas, geomorfológicas e hidrológicas, es un país expuesto a la ocurrencia de desastres, definidos según la Comisión Económica para América Latina y el Caribe (CEPAL, 2014), como la combinación de fenómenos naturales y la vulnerabilidad de las personas o asentamientos humanos. Además, los desastres pueden dejar innumerables daños físicos, económicos, sociales y emocionales en las comunidades aledañas a estos, por ejemplo, según la Unidad Nacional para la Gestión del Riesgo de Desastres (UNGRD, 2018), entre abril de 2017 y abril de 2018, el número de personas afectadas por desastres naturales es de 603.302 habitantes. Es necesario considerar que las condiciones medioambientales y sociopolíticas de las regiones aumentan los factores de riesgo, es decir, la vulnerabilidad y la amenaza de las comunidades, términos que presentan diferentes nociones y abordajes teóricos.

Ahora bien, algunos autores señalan la evolución de concepciones en materia de catástrofe o desastres, haciendo especial énfasis en considerar los desastres como sucesos o situaciones de carácter colectivo, que involucran la presencia de daños de diferente orden, es decir, físicos, humanos, medioambientales o económicos. Adicionalmente, se presentan grandes retos al momento de abordar desde la investigación estos conceptos, debido que su experiencia corresponde, por un lado, a las instituciones y, por otra parte, a la experiencia y subjetividad de las personas o colectivos afectados (Arcos \& Castro, 2015).
Duque (2019) presenta una distinción entre las nociones de riesgo, vulnerabilidad y amenaza. Según el autor, el riesgo se encuentra asociado a la probabilidad de que un evento de carácter natural se presente en un sitio específico y en un tiempo determinado, mientras que la vulnerabilidad se entiende como un factor de riesgo que incluye la exposición de las vidas y en general de los bienes ante un fenómeno natural y, a su vez, depende de factores sociales, culturales, educativos, ambientales, físicos, económicos y políticos (Departamento Nacional de Planeación DPN, 2018). Por su parte, el concepto de amenaza se determina como una función propia de la susceptibilidad, el fenómeno detonante y el potencial de energía. Sin embargo, otros autores señalan que los factores ambientales del riesgo o de la vulnerabilidad pueden analizarse como amenaza o desastre (Caicedo, 2013).

Otro elemento para considerar es el impacto que presentan los desastres sobre la salud mental comunitaria, por cuanto las personas experimentan una serie de sensaciones de pérdida de bienes materiales, familiares y sociales y que a largo plazo se podrían convertir en factores de riesgo que desencadenan en trastornos de ansiedad, estrés post traumático, depresión, problemas gástricos, cefaleas y dificultades cardíacas, entre otros. Según la Organización Mundial de la Salud (OMS), estas situaciones se consolidan como una carga sobre la salud mental de las personas que, por lo general, poseen estrategias de afrontamiento o mecanismos de respuesta limitados para sobrellevar las dificultades. Sumado a esto, la mayoría de personas que viven estas situaciones se encuentran entre los estratos económicos más bajos. De igual manera, interrumpen sus actividades 
cotidianas, contribuyendo al desajuste negativo frente al impacto psicológico (Albedaño \& Fernández, 2016).

Por su parte, en la ciudad de Pasto las comunidades aledañas al volcán Galeras han desarrollado un entramado de ideas, creencias y percepciones sobre la influencia que presenta el volcán en la vida de sus habitantes y que se entiende como el legado histórico que dejaron sus ancestros. Tras la investigación realizada por Jurado, Ojeda y Ojeda (2018), la cual tuvo como objetivo analizar la presencia de un volcán activo y los sentimientos, pensamientos y significados que se configuran en las personas que habitan en cercanías, se determinó que las representaciones sociales de la comunidad con respecto al volcán Galeras, cumplen funciones identitarias y de orientación entre sus habitantes. Asimismo, un estudio desarrollado por López y Ojeda (2017) enfatizó en la importancia de la tradición oral entre generaciones, pues éstas poseen memorias colectivas relacionadas con la experiencia de vida de sus antepasados en referencia al volcán y que, al mismo tiempo, fortalecen la construcción social de las realidades y el futuro de las nuevas generaciones. No obstante, y tras la revisión de antecedentes, no se evidenciaron estudios que logren identificar y analizar el papel de la resiliencia comunitaria, especialmente en la comunidad de Mapachico, lo que determina la importancia de los avances en el proceso investigativo.

La comunidad de Mapachico Centro pertenece al corregimiento de Mapachico, el cual se encuentra ubicado a las faldas del volcán Galeras, en el noroccidente de la cabecera municipal de Pasto, específicamente a $7 \mathrm{~km}$ de la ciudad. A su vez, las creencias en torno a los procesos de convivencia con el volcán y que, además, caracterizan a los habitantes de la comunidad de Mapachico Centro, presentan elementos desde su cosmovisión y sentido de comunidad (Dorado, Hernández \& Ruano, 2019), ya que en muchas ocasiones describen su relación con la naturaleza como armónica. Asimismo, el imaginario colectivo de los habitantes de Mapachico Centro se encuentra interiorizado, puesto que el volcán Galeras es considerado como un padre, que puede dar vida y, a la vez, arrebatarla, e incluso, suelen atribuir explicaciones mágicas al fenómeno natural.

Sin embargo, en la actualidad, la comunidad de Mapachico Centro se enfrenta a un problema de orden constitucional, pues en el año 2005 la Presidencia de la República declaró la existencia de una situación de desastre en el municipio de Pasto, como consecuencia de la presencia y actividad del volcán, del cual la comunidad de Mapachico Centro se constituye como uno de los sectores más afectados. Más adelante, el decreto 3905 de 2008 establece el Plan de Reasentamiento en la Zona de Amenaza Volcánica del Volcán Galeras, generando una serie de inequidades sociales en materia de inversión estatal, pero, lo más importante, perjudicando la dinámica comunitaria y el tejido social de la comunidad.

En el mes de junio de 2018, la comunidad de Mapachico Centro experimentó una serie de situaciones conflictivas a causa de los sismos de influencia volcánica, los cuales cobraron la vida de 2 personas y más de 90 familias damnificadas, distribuidas entre las diferentes veredas del corregimiento de Mapachi$\mathrm{co}$, especialmente las veredas de Mapachico Centro y Briceño (UNGRD, 2018). 
Además, los problemas de salud mental de los habitantes de la comunidad fueron significativos, debido a la interrupción de su cotidianidad. La mayoría de sus habitantes se ubicaron en albergues improvisados por un periodo aproximado de un mes (tiempo superior al sugerido en casos de desastres), que, si bien estaban instalados, presentaban un deterioro físico considerable, generando un malestar social y contribuyendo al desajuste psicológico por parte de los habitantes.

En el marco de la investigación se hace énfasis en los pilares de la resiliencia comunitaria, propuestos desde el modelo de Suárez (2001), las cuales ponen en evidencia los recursos psicosociales y comunitarios con los que cuenta o desarrolla una determinada comunidad, luego de enfrentarse a situaciones traumáticas, en este caso, las consecuencias que dejan los desastres de índole sísmico en la comunidad de Mapachico Centro. Además, según Flores y Sanhuesa (2018), el análisis cualitativo de la resiliencia es imprescindible en la interpretación de factores protectores comunitarios como fuentes de transformación y bienestar social (Calle, 2010).

Para la comprensión teórica se propone el abordaje de las siguientes dimensiones que forman parte del proceso investigativo y se describen a continuación:

\section{Resiliencia Comunitaria}

A diferencia de los modelos clásicos que explican la resiliencia desde la particularidad o una característica de la personalidad (Wagnild \& Young, 1990, citados en Montalvo \& Reyes, 2019), es decir, que se refiere a la resiliencia como una condición personal para encontrar oportunidades en la tragedia (Menanteux, 2015). El modelo propuesto por Suárez (2001) define la resiliencia comunitaria como una serie de condiciones colectivas que tienen como propósito sobreponerse a situaciones que impliquen desastres o situaciones masivas de adversidad y, a partir de ello, reconstruir los procesos. De igual forma, el estudio desarrollado por el autor reconoce la presencia de los pilares de la resiliencia comunitaria, los cuales son: 1. Autoestima colectiva, que involucra la satisfacción por la pertenencia a la comunidad; 2. Identidad cultural, como proceso integral de valores, costumbres, sentimientos y otras expresiones artísticas y culturales, que aumentan el sentido de pertenencia; 3. Humor social, como la capacidad de incluir la comedia en las situaciones o adversidades de carácter colectivo; y 4. Honestidad estatal, vista como la resistencia a los procesos que afecten el tejido y desgastan la dinámica de integración social entre las comunidades.

Ahora bien, los anteriores pilares permiten consolidar que el problema de estudio de la investigación se encuentra determinado por dos aspectos. El primero de ellos es el reconocimiento del vacío de conocimiento, en materia de investigaciones, que den cuenta de los procesos de resiliencia comunitaria en el departamento de Nariño y, específicamente, en la comunidad de Mapachico Centro. El segundo corresponde a la carencia de estudios de resiliencia comunitaria desde el modelo de los pilares de la resiliencia comunitaria de Suárez (2001), pues es una propuesta creada desde los pueblos latinoamericanos. 


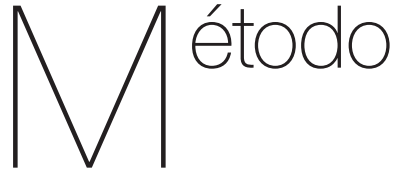

El presente artículo de investigación se desarrolló a través de la implementación de las consideraciones epistémicas del paradigma cualitativo (Pita \& Pértegas, 2002), debido a su riqueza metodológica y su carácter inductivo. El enfoque que se empleó es el fenomenológico, ya que, como lo afirma Rusque (2001), representa un conjunto de interpretaciones significativas de la realidad, desde la cotidianidad de las personas, reconociendo las posibles relaciones fenomenológicas (Mari, Bo Bonet \& Climent, 2010.) Asimismo, el uso del enfoque etnometodológico enriqueció el proceso, ya que se analizaron las prácticas o métodos empleados por los participantes en situaciones particulares y que les permitieron dar un sentido del orden social, de forma endógena (Van, 2011).

\section{Participantes/Unidad de Análisis}

Se contó con la participación de 18 residentes de la comunidad de Mapachico Centro y 3 funcionarios públicos que lideran procesos de desarrollo comunitario, entre 30 y 70 años (35\% de hombres $65 \%$ de mujeres). Como criterios de inclusión se establecieron: 1. Haber nacido en la comunidad de Mapachico Centro; 2. Formar parte del registro de los procesos de la Junta de Acción Comunal (JAC) o el Cabildo Indígena de Mapachico; 3. Ser mayor de edad y 4. Residir en la comunidad durante los últimos 20 años.

\section{Técnicas e Instrumentos de recolección de información}

Revisión documental: esta técnica fue indispensable durante el proceso, pues su aporte proporcionó información relevante del reconocimiento y consolidación de la problemática del estudio, los referentes teóricos e investigativos que conformaron los ejes de estudio (Valencia, 2015).

Entrevista a profundidad: se realizaron 5 entrevistas a profundidad con los líderes de la comunidad, facilitando el acercamiento entre los investigadores y los participantes (Monje, 2011). Además, para su elaboración, y sin dejar a un lado su flexibilidad metodológica, se retomaron las categorías sugeridas por el modelo de resiliencia de Suárez (2006), específicamente autoestima colectiva e identidad cultural.

Observación participante: su implementación se logró a lo largo de todo el proceso investigativo, ya que permitió identificar el entramado de ideas, experiencias y representaciones simbólicas (Jociles, 2008) de los habitantes en torno a los procesos de convivencia con el volcán y las consecuencias de los desastres.

Grupo focal: se ejecutaron 3 grupos focales, en los que participaron 6 personas respectivamente y brindó la posibilidad de recolectar información desde las percepciones de cada habitante, así como de los funcionarios públicos y los investigadores, a través del debate en conjunto (Hamui-Sutton \& Varela-Ruiz, 2013) discutiendo temáticas relacionadas 
con las categorías humor social y honestidad estatal (Suarez, 2001).

\section{Procedimiento}

Teniendo en cuenta que el proceso investigativo se proyectó desde el paradigma cualitativo y el enfoque fenomenológico, Narváez (2019) sugiere la consolidación de algunas fases, las cuales se retoman a continuación:

Fase de contacto: Se realizaron encuentros de acercamiento entre los líderes de la comunidad y también con los funcionarios públicos, pues todos ellos presentaron una apertura y se mostraron interesados en el proceso investigativo.

Fase de inserción participativa: luego de realizar algunos acercamientos con los líderes de la comunidad, especialmente con los miembros de la JAC, se prosiguió a gestionar la autorización de las instituciones involucradas, como la Universidad de Nariño y la Alcaldía de Pasto, con el objetivo de permitir la asistencia de los investigadores a las reuniones locales, asambleas y mingas comunitarias.

Fase de recolección de información: posterior a la elaboración de las técnicas e instrumentos que fueron revisadas por tres expertos quienes, mediante las sesiones previas, determinaron el cumplimiento de éstas. Con base en los criterios de claridad de los objetivos, dimensiones de investigación, sustento teórico, estructura de los métodos y el impacto esperado, se prosiguió a realizar los respectivos ajustes para finalizar con la aplicación de cada una de acuerdo con el cronograma establecido.

Fase de análisis de información: según los datos recolectados se instauró el plan de análisis, el cual consistió en la reducción y organización de la información y emisión de conclusiones desde la elaboración de unidades de significado general y la consolidación del informe final. Para ello, se establecieron las macrocategorías y subcategorías, según Spradley (1980). Además, fue necesario generar códigos que favorecieron la identificación de las frases o expresiones, mediante la codificación abierta, la cual contiene la identificación de los participantes así como de las iniciales de la técnica desarrollada. Por ejemplo: sujeto 1 (S1) entrevista a profundidad (EP.)

Al finalizar se contrastaron los resultados obtenidos con los referentes alusivos y se crearon las conclusiones (Rodríguez, Quiles \& Herrera, 2005). Para una mayor comprensión, la Tabla 1 presenta el consolidado de las categorías según las técnicas e instrumentos empleados.

Tabla 1.

Consolidado de categorías según técnicas e instrumentos

\begin{tabular}{|c|c|c|c|}
\hline Técnicas & Instrumentos de recolección & Macrocategorías & Subcategorías \\
\hline Revisión documental & Matriz de consistencia & \multirow{4}{*}{$\begin{array}{l}\text { Autoestima colectiva. } \\
\text { Identidad cultural. } \\
\text { Humor social. } \\
\text { Honestidad gubernamental. }\end{array}$} & \multirow{4}{*}{$\begin{array}{l}\text { Participación comunitaria. } \\
\text { Convivencia. } \\
\text { Sensibilidad ambiental. }\end{array}$} \\
\hline Entrevista a profundidad & Guión de entrevista & & \\
\hline Observación participante & Diario de campo & & \\
\hline Grupo focal & Guión de grupo focal & & \\
\hline
\end{tabular}

Fuente. Propia. 


\section{Consideraciones éticas}

La investigación se desarrolló en el marco de los principios y consideraciones de la resolución 8430 de 1993 (Ministerio de Salud, 1993), Título II, de la investigación con seres humanos, teniendo en cuenta las siguientes recomendaciones: 1. Respeto por la dignidad, protección de los derechos y el bienestar; 2. Ajuste a los principios éticos y científicos; 3. Seguridad de las personas que conformaron el proceso investigativo; 4. Cuenta con el Consentimiento informado y por escrito de los miembros de la comunidad, y organizaciones comunales como la Junta de Acción Comunal (JAC); y 5. El procedimiento está a cargo de profesionales, expertos en el tema de estudio, así como también se contó con la supervisión del presidente de la JAC.

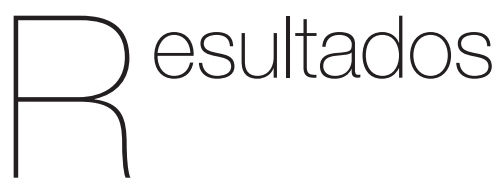

A continuación, se presentan los resultados obtenidos a través de las técnicas e instrumentos desarrollados, los cuales se describen a partir de la consolidación de las categorías establecidas por el modelo de resiliencia de Suárez (2001) y las categorías emergentes que enriquecieron el proceso investigativo.

\section{Autoestima colectiva}

El proceso investigativo evidenció que la comunidad de Mapachico Centro ha construido a lo largo de sus 70 años, una serie de elementos que determinan la consolidación de factores protectores en salud y elementos interculturales, los cuales están basados en la constitución de un sistema que, desde la cosmovisión de los habitantes de la comunidad de Mapachico Centro, responde a los procesos de identificación del corregimiento. En este sentido, se encuentra que los habitantes han forjado su territorio alrededor del imaginario que tienen con la presencia del volcán Galeras, pues éste les ha brindado un territorio fértil y pacífico.

Además, la identificación con el territorio les ha creado un vínculo especial con lo que ellos denominan como su casa, pero sobre todo con la comunidad de Mapachico Centro, pues, a partir de las catástrofes naturales ocurridas en el año 2018, han surgido una serie de comportamientos y actitudes a favor del otro, resaltando el cooperativismo, la solidaridad, la empatía y la resolución de conflictos. Muestra de esto se puede evidenciar en el siguiente relato:

"Cuando pasó lo del volcán nos sentimos más unidos que nunca, pues ver a la vecina o el vecino con la casita derrumbada y sin saber qué hacer, me partió el alma y uno los conoce desde hace mucho tiempo, cómo uno no los va a poder ayudar, aunque sea ayudándoles a rescatar las cositas y brindándoles un hombro así sea para que lloren y se desahoguen" (S4EP).

Además, se considera que los factores protectores en salud mental comunitaria, de los cuales forma parte la autoestima colectiva favorece su visión positiva del futuro, por lo que la proyección de sus habitantes estuvo marcada por el anhelo 
de salir adelante a pesar de las adversidades, aunque es evidente que los efectos que dejan las catástrofes naturales, no solo se consolidan en aspectos positivos, sino que, producto de la identificación y el colectivismo, dan pie a generar un malestar y división interna por parte de los grupos organizados, como la JAC y la Oficina Corregimental. A continuación, se presentan dos relatos que sustentan este análisis:

"A pesar de que todos somos del corregimiento, siempre están los que por preferencias ayudan a los que les conviene" (S3EP). "En la Junta de Acción Comunal, que se hizo, pues ayudar a los que ellos consideraban del grupo, mientras que el resto nada (S1EP). Mire los viejitos, si no es por uno que les aconseja que vayan y soliciten ayuda, si no se quedan sin nada" (S2EP).

\section{Identidad cultural}

El papel que juega la cosmovisión y la constitución de la identidad cultural son aspectos que han formado parte de la construcción sociohistórica de la comunidad, pues toman como referente la ubicación geográfica y su cercanía con el volcán Galeras. En la mayoría de los discursos encontrados se identificó que las consecuencias de los desastres ocurridos son el producto del malestar del volcán y por las decisiones políticas que se han tomado, especialmente desde el gobierno central; se consolida como un pilar identitario en el común de las personas, y que, además, responde a la visión de un pueblo que desde sus orígenes resistió contra otras comunidades.
Por consiguiente, se presentan relatos que respaldan esta aseveración:

"A menudo nuestro papito (Volcán) se pone bravo, porque no está de acuerdo con lo que nos han hecho como pueblo, pues si aquí, todos hemos nacido en este territorio $y$ moriremos aquí, nadie nos va a sacar, ya sea porque la muerte nos encuentre o porque nos mate el Volcán" (S1EP).

"Sí me asuste cuando comenzaron los temblores, pero yo pienso en que todos estamos acostumbrados a vivir con esos temblores" (S1EP).

Otro factor de la identidad cultural es el conjunto de prácticas y saberes que se han construido desde los orígenes, pues, si bien no hay documentos que determinen la veracidad de la constitución del corregimiento como zona habitada por indígenas o resguardos, muchos presentan características compartidas de los pueblos ancestrales. Es el caso de la conformación del Cabildo Indígena de Mapachico, el cual, a pesar de que no se encuentra constituido ante el Ministerio del Interior, se ha posicionado como un organismo de desarrollo social y de gestión comunitaria.

No obstante, el análisis de la identidad cultural propone tener una visión integral de los habitantes, ya que se evidencia que existen divisiones comunitarias entre los sectores campesinos y los indígenas; pues ambos responden a diferentes visiones de lo que es el territorio y su comprensión. Ahora, dentro de la dinámica comunitaria y relacionada con los procesos de resiliencia, se logró analizar que la catástrofe natural permitió la unión 
de todos los habitantes, sin importar su origen o sus creencias, por lo que el sentimiento de unidad y también de otredad disminuyó en las épocas posteriores a la catástrofe. A continuación, se muestra un relato que afirma esta unidad:

"Cuando uno va y ayuda al otro no está mirando su origen, si es indígena o campesino, da igual, lo importante es que todos trabajemos por mejorar nuestros hogares, me duele que en algunas personas no se comprenda esta idea, pero bueno, no por eso la voy a dejar a un lado" (S7EP).

\section{Humor social}

Esta categoría no se expresó de manera explícita en los habitantes, puesto que se considera que dada la proximidad de los eventos es un recurso que se construye a futuro. Sin embargo, algunos elementos dieron cuenta de cómo las estrategias de afrontamiento basadas en la capacidad de incluir la comedia como proceso resiliente configuró el pensamiento de las personas con relación a los vínculos con el volcán. Asimismo, más allá de determinar si se presentó o no la comedia, algunas expresiones identificadas como el tono de la voz, los gestos y sobre todo las actitudes dieron a conocer las creencias que probablemente se asocien como elementos de la comedia. A continuación, se encuentran comentarios relacionados con esta categoría:

"El volcán se enojó con nosotros (se perciben risas) porque el gobierno no está ayudando a la comunidad (S5EP)". "El Galeras está jbravísimo! (expresión de sorpresa) porque votamos basura, usamos sus tierras sin control" (S2EP). "Al volcán no le gusta que lo molesten en las fiestas, por eso ruge (expresión de asombro) y nos hace estremecer para que nos demos cuenta que él es nuestro padre" (S4EP).

\section{Honestidad gubernamental}

El descontento social con respecto a temas relacionados con la gestión gubernamental nacional reafirma la consolidación de procesos de resistencia comunitaria ante las disposiciones nacionales sobre la declaración del corregimiento como zona de amenaza volcánica y el proceso de reasentamiento. Adicionalmente, los miembros de la comunidad presentan actitudes desfavorables frente a las acciones del gobierno central. Consideran que tanto la declaración de zona de desastre y el plan de reasentamiento no cuentan con la evaluación integral de las necesidades psicosociales de los habitantes y, por el contrario, son estrategias que afectan el tejido y la integración social. Asimismo, la inversión estatal sobre estas zonas es menor en comparación con otras comunidades. Estas visiones se comparten en todos los habitantes y, como muestra de ello, han surgido acciones colectivas como manifestaciones, bloqueo de vías de acceso y mingas de pensamiento. Este análisis se basó en los siguientes comentarios:

"El gobierno nacional solo inos ha mentido! (se percibe enojo), no tiene en cuenta nuestras necesidades ni reconoce nuestro territorio" (S6GF1). "Si le dijera cuantos de los altos mandos han venido a 
Mapachico, le diría que ninguno, (expresión de descontento), pues para ellos es fácil coger y declarar una zona como de desastre, pero no saben las consecuencias que tienen para nosotros este tipo de decisiones" (S2GF3). "Como quiere que la gente crea en el gobierno o en el presidente, si este solo emite una resolución que no tiene nada que ver con lo que se vive" (S3GF2).

Además de las categorías evidentes en el modelo propuesto para analizar la resiliencia comunitaria, el proceso investigativo arrojó dos categorías emergentes que son importantes incluir en los resultados, a saber:

\section{Participación Comunitaria}

La resiliencia comunitaria no solo da cuenta de los recursos o el estado de la comunidad ante las consecuencias de un desastre natural, sino que también consolida el grado de acción y participación comunitaria respecto a desarrollar comités de ayuda en casos de emergencias. Para ello, se presentan los siguientes relatos:

"lo importante es que todos nos organizamos para ayudar a los demás, unos se encargaban de recibir las ayudas de las empresas, otros ayudaban con el censo de damnificados y otros repartían las ayudas" (S2GF1). "La JAC determinó la creación de un comité de emergencias que ayude no solo con los recursos que llegaban de las empresas sino a transmitir información oficial" (S4GF2).

\section{Sensibilidad ambiental y convivencia}

En la comunidad es clara la conexión que presentan con la naturaleza, puesto que, para muchos la constituyen como la madre o la casa donde habitan; por esta razón, el proceso investigativo sugirió presentar esta categoría como emergente, ya que, dadas las condiciones en las que se presenta la resiliencia comunitaria, corresponden al desconocimiento de la comunidad sobre la importancia de las acciones medioambientales que se pueden gestar para evitar problemáticas subyacentes de los eventos sísmicos. Además, el estudio sugiere que dentro de las catástrofes ambientales se deben emplear planes o estrategias de convivencia y educación continua para las comunidades.

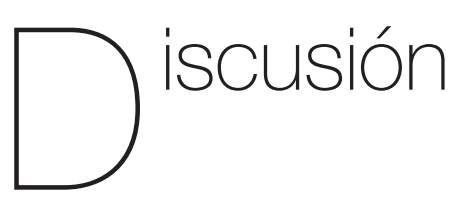

A partir de los resultados obtenidos se realizó una aproximación fenomenológica a los procesos de construcción de la resiliencia comunitaria dentro de la comunidad de Mapachico Centro. Estos se han constituido como el resultado de una serie de elementos que se caracterizan por su dinamismo y que de acuerdo con Jurado et al. (2018), la cosmovisión de los habitantes cumple una función identitaria, debido a la convivencia y la familiarización que presentan con el volcán Galeras, ya que configuran el imaginario colectivo y las representaciones sociales. De igual manera, se evidenció dentro de las experiencias y sentires comunitarios, que 
la percepción de desastre natural se encuentra relacionada con el planteamiento de Chaux (1993, citado en Albedaño \& Fernández, 2016), quien refiere que la aparición de un fenómeno natural no necesariamente provocará un desastre natural, comprendido, no solo como los daños materiales o la pérdida de la vida, sino que además incluye la percepción de la comunidad frente a la vulnerabilidad y el propio riesgo.

Se comprobó que en los habitantes, si bien atribuyen explicaciones desde la cosmovisión y la dialéctica (López \& Ojeda, 2017), existe una interrupción de la dinámica cotidiana evidente de la comunidad, puesto que los eventos sísmicos, así como las réplicas, las cuales se extendieron por el periodo de un mes (aproximadamente), obligaron a las personas a desocupar sus viviendas, generando un malestar psicológico colectivo y contribuyeron al desajuste socioeconómico (OMS, 2002, citado en Albedaño \& Fernández, 2016), es decir, a menudo se percibía el entorno como amenazante; por eso, la mayoría tuvieron que reubicarse en albergues temporales, donde las condiciones para habitar no eran suficientes o no estaban diseñados para emplearse por periodos extensos.

Además, estas consideraciones determinan la importancia de la salud mental comunitaria como la acción contingente y proactiva de los factores protectores como los pilares de la resiliencia (Flores \& Sanhuesa, 2018), los cuales, les permitieron hacer frente a situaciones difíciles y entre los que la comunidad destaca el uso de valores sociales como la solidaridad, la empatía y el respeto. De igual manera, presentan una estrecha relación con los pilares de la resiliencia, pues a menudo se reconocen como parte de la autoestima colectiva, la identidad cultural y el humor social, siendo elementos propios de los habitantes de la comunidad de Mapachico Centro, así como también la construcción y el fortalecimiento del sentido de comunidad, presentando características similares con las categorías de honestidad gubernamental e identidad cultural, en función de los sentimientos de pertenencia hacia los planes y acciones colectivas, lo que reafirma las consideraciones de Dorado et al. (2019).

Para reconocer el verdadero proceso resiliente $\mathrm{y}$, en concordancia con Calle (2010), quien afirma que el análisis cualitativo de las estrategias o recursos de afrontamiento comunitario, constituyen el eje central de la resiliencia, se encontró que en la comunidad de Mapachico Centro, el proceso de resiliencia comunitaria puede estar ligado a la percepción que tienen de comunidad, puesto que, para ellos, no solo es el compartir un espacio físico, sino que, además, la identificación con éste responde a la construcción sociohistórica de sus habitantes y sus ancestros (López \& Ojeda, 2017). A su vez, los procesos socio-cognoscitivos de la comunidad se encuentran determinados, en parte, por los postulados de Sánchez (2001, citado en Musitu, Herrera, Cantera \& Montenegro, 2004), quien afirma que en la búsqueda de objetivos colectivos las comunidades fomentan la cooperación y dejan a un lado cualquier diferencia. Por su parte, la definición de Montero (2004) aporta elementos significativos en función del desarrollo emergente de procesos de organización y participación comunitaria como comités y organizaciones que ayudaron a gestionar recursos y brindaron apoyo emocional. 
La aproximación a la resiliencia comunitaria se identifica en primer lugar, desde el modelo de Suárez (2001), ya que corresponde a la construcción colectiva de condiciones comunitarias como recursos que les permitieron enfrentarse a situaciones difíciles; en segundo lugar se identificó que la construcción colectiva no necesariamente es excluyente de la particularidad de sus habitantes (Wagnild \& Young, 1990, citados en Montalvo \& Reyes, 2019), en el sentido de que cada uno ha construido formas particulares de vivir y expresar la comunidad.

Ahora bien, la relación entre los pilares de la resiliencia comunitaria, según el modelo de Suárez (2001), evidenciaron lo siguiente: 1. La autoestima colectiva no solo refiere la satisfacción de pertenecer a la comunidad, pues en ésta es importante el papel que presentan los elementos sociohistóricos de la comunidad; 2. Probablemente la identidad cultural sea el componente en el que se encontró mayor información puesto que la comunidad presenta una riqueza cultural e histórica significativa con respecto a otras comunidades; 3. El humor social no se evidenció de manera significativa, lo que sugiere que en la comunidad no se identifica esta característica desde la cotidianidad, ya que para ellos es una forma de irrespeto y ausencia de solidaridad con el resto de la comunidad; y 4. El componente de honestidad estatal es el pilar que mayor diferencia presenta a nivel organizativo en los habitantes, ya que, muchos habitantes rechazan las acciones del gobierno central, pues consideran que las decisiones no cuentan con la suficiente comprensión social y cultural de los habitantes, sobre todo en temas de reasentamiento y compra de predios.

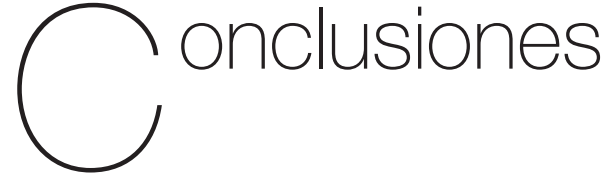

Es importante concluir que el presente estudio cumplió con el objetivo de analizar el papel de la resiliencia comunitaria frente a los desastres en un grupo de habitantes de la comunidad de Mapachico Centro de la ciudad de San Juan de Pasto, puesto que se determinó que la resiliencia, si bien es un recurso que se encuentra en la comunidad, se activa de forma contingente a las catástrofes o desastres, puesto que éstas movilizan diferentes recursos y estrategias para hacer frente a las necesidades. Asimismo, cada uno de los miembros de la comunidad concluye que los eventos sísmicos, y en sí la convivencia, les han permitido reconocer el valor de la unión comunitaria, no como un instrumento asistencialista sino de empoderamiento social.

Se resalta la importancia del estudio de la resiliencia desde la comprensión de marcos compresivos y teóricos que permitan analizar de manera sistémica los componentes; además, el modelo de resiliencia comunitaria de Suárez (2001), reconoce el proceso resiliente como una particularidad de las comunidades, por lo que su idoneidad paradigmática favoreció la consecución de la investigación. Es probable que la resiliencia comunitaria se encuentre ligada a identidades culturales que, desde la cosmovisión de los habitantes de la comunidad, facilitan y direccionan las acciones investigativas.

La importancia de implementar estudios cualitativos en la comunidad es un factor imprescindible, pues reconoce el valor humano y cambia la perspectiva 
del trabajo con comunidades, ya que fue necesario adentrarse en la comunidad para reconocer elementos culturales que fueron el punto de partida para explicar la comprensión de fenómenos como la salud mental y la resiliencia. Además, el estudio se constituye desde la construcción participativa de sus habitantes, puesto que estos fueron la fuente de conocimiento y exploración, lo cual fue enriquecedor en los procesos de familiarización, recolección de datos y análisis, promoviendo actitudes de empoderamiento y generando la interacción social.

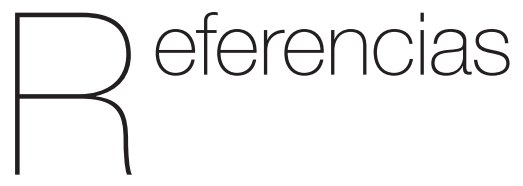

Albedaño, R. \& Fernández, R. (2016). Salud Mental en la comunidad en situación de desastre. Una revisión de los modelos de abordaje de la comunidad. Ciência \& Saúde Coletiva, 21(2), 431-442.

Arcos, P. \& Castro, R. (2015). La construcción y evolución del concepto de catástrofedesastre en medicina y salud pública de emergencia. Revista INDEX de enfermería, 2(1-2), 59-61.

Caicedo, A. (2013). HABITAR BAJO RIESO (Trabajo de Grado de Magister). Facultad de Arquitectura. Universidad Nacional, Medellín.

Calle, A. (2010). Apego, desarrollo y resiliencia. Informes Psicológicos, 12(1), $25-40$
Comisión Económica Para América Latina y el Caribe CEPAL (2014). Manual para la evaluación de desastres (Informe Técnico). Chile: Naciones Unidas.

Decreto 3905. Plan de Reasentamiento en la Zona de Amenaza Volcánica Alta (ZAVA). Del Volcán Galeras. Ministerio del Interior y de Justicia, 07 de octubre de 2008.

Decreto 4106. Por el cual se declara la existencia de una situación de desastre en los municipios de Pasto, Nariño y La Florida, en el Departamento de Nariño. Ministerio del Interior y de Justicia, 15 de noviembre de 2005.

Departamento Nacional de Planeación DPN, (2018). Índice Municipal de Riesgo de Desastres de Colombia. (Informe digital). Colombia: Departamento Nacional de Planeación.

Dorado, A., Hernández, M., \& Ruano. F. (2019). La evaluación comunitaria: una herramienta de integración social en los habitantes de la comunidad Arcoíris. Psicoespacios, 13(22), 40-61.

Duque, G. (2019). Informe Gestión del riesgo natural y el caso de Colombia (Informe de Gestión). Colombia: Banco Mundial de Colombia.

Flores, P. \& Sanhueza, R. (2018). Resiliencia comunitaria frente a desastres naturales: caleta Tumbes, región del Biobío, Chile. Revista Cuadernos de Geografía, 27(1), 131-145.

Hamui-Sutton, A. \& Varela-Ruiz, M. (2013). La técnica de grupos focales. Investigación en Educación Médica, 2(5), 55-60. 
Jociles, M. (2018). La observación participante en el estudio etnográfico de las prácticas sociales. Revista Colombiana de Antropología, 54(1), 121-150.

Jurado, N., Ojeda, E., \& Ojeda, N. (2018). Representaciones sociales en una comunidad educativa de la Zona de Amenaza Alta del Volcán Galera. Boletín de Geología, 3(3), 195-210.

López, E. \& Ojeda. D. (2017). Relaciones intergeneracionales en la construcción social de la percepción del riesgo. Desacatos, 54, 106-121.

Mari, R., Bo Bonet, R., \& Climent, C. (2010). Propuesta de análisis fenomenológico de los datos obtenidos en entrevista. Ciencias de la Educación, 8(1) 113-133.

Menanteux,M.(2015). Resilienciacomunitaria. Abordaje teórico y vinculación al ejercicio de la psicología comunitaria en el contexto latinoamericano actual (Tesis de Maestría). Facultad de Ciencias Sociales. Universidad de Chile, Santiago de Chile.

Ministerio de Salud, 1993. Resolución 8430. Por la cual se establecen las normas científicas, técnicas y administrativas para la investigación en salud, 4 de octubre de 1993.

Monje, C. (2011). Guía didáctica Metodología dela Investigación. Colombia: Documento digital. Universidad Surcolombia.

Montalvo, L. \& Reyes-Bossio, M. (2019). Resiliencia y Autoconcepto Personal en indultados por terrorismo y traición a la patria residentes en Lima. Informes Psicológicos, 19(1), 119-140. http://dx.doi. org/10.18566/infpsic.v19n1a07
Montero, M. (2004). Introducción a la psicología comunitaria. Desarrollo, conceptos y procesos. Argentina: Primera Ed. Paidós.

Musitu, G., Herrero, J., Cantera, L., \& Montenegro, M. (2004). Introducción a la Psicología Comunitaria. España: Primera Ed. UOC.

Narváez, J. (2019). Violencia y fronteras imaginarias en la ciudad. Colombia: Universidad de Nariño.

Pita, S. \& Pértegas, S (2002). Investigación cuantitativa y cualitativa. Cuadernos de Atención Primaria, 9, 76-78.

Rodríguez, C., Quiles, O., \& Herrera, L. (2005). Teoría y práctica de análisis de datos cualitativos. Proceso general y criterios de calidad. Revista Internacional de Ciencias Humanas, 15(2), 133-154.

Rusque, A. (2001). De la diversidad a la unidad en la Investigación Cualitativa. Venezuela: Tercera Ed. Vadell Hermanos.

Suárez, E. (2006). Resiliencia y Subjetividad. Los Ciclos de la Vida. Argentina: Primera Ed. Paidós/Tramas Sociales.

Spradley, J. (1980). Observación Participante. España: Santillana.

Suárez, N. (2001). Una concepción latinoamericana: la resiliencia comunitaria. Argentina: Paidós.

Unidad Nacional para la Gestión del Riesgo de Desastres (2018). Colombia desastres naturales (Informe Técnico). Colombia: Oficina de Naciones Unidas para la Coordinación de Asuntos Humanitarios. 
Valencia, V. (2015). Revisión documental en el proceso de investigación Univirtual. Colombia: Documento digital.
Van, T. (2011). Sociedad y Discurso. Literatura y Lingüística, 4(3), 287-292. 